Carcinogenesis

\title{
Anthraquinone laxatives and human cancer: an association in one case
}

\author{
P.M. Patel, P.J. Selby, Judith Deacon, Clair Chilvers and T.J. McElwain
}

Sections of Epidemiology and Medicine, Institute of Cancer Research, Royal Marsden Hospital, Downs Road, Sutton, Surrey, UK.

\begin{abstract}
Summary: An 18 year old girl presented with a leiomyosarcoma of the small bowel with widespread dissemination. Despite short term remissions after chemotherapy and surgery she died of the disease 10 months later. A history of prolonged exposure to danthron (an anthraquinone laxative) in childhood was obtained. Anthraquinones are known to be mutagenic and may be carcinogenic in experimental systems but danthron is not a proven carcinogen in man. The association of danthron and a rare bowel cancer in one case cannot prove a causative link. However, collection of further data seems advisable.
\end{abstract}

\section{Introduction}

Natural and synthetic anthraquinones are widely spread in our environment being used in food, cosmetics, hair dyes, textiles and drugs. Probably the commonest pharmaceutical application is in laxatives. Anthraquinone laxatives include danthron and its glycosides which are to be found in senna and cascara. Danthron, (also referred to as 1,8-dihydroxyanthraquinone, 1,8-dihydroxy-9,10-anthracenedione or chrysazin and marketed in the combination laxatives Dorbanex and Normax) is mutagenic in Salmonella typhimurium. ${ }^{1,2}$ There is some evidence that it is carcinogenic in rodents. ${ }^{3,4}$ Available epidemiological studies have not shown any link between anthraquinones and cancer in man. ${ }^{5}$

We report here a single case in which a small bowel sarcoma occurred at age 18 years in a girl who had had prolonged exposure in early childhood to a combination laxative containing danthron.

\section{Case report}

An 18 year old Caucasian girl presented as an emergency with acute lower abdominal pain. This had been preceded by several weeks of intermittent colicky pain with occasional vomiting and constipation. An emergency laparotomy was performed and a small bowel tumour was found in the distal ileum. The primary tumour was $8 \mathrm{~cm}$ in diameter and secondary deposits were palpated in the omentum and the

Correspondence: Professor P. Selby, M.D., M.R.C.P.

Accepted: 14 December 1988 mesenteric lymph nodes. The small bowel tumour was resected and she made a good recovery.

Pathological examination revealed a haemorrhagic and necrotic tumour forming a polypoid mass projecting into the lumen of the bowel. The tumour was composed of large, plump spindle cells with vesicular nuclei and prominent nucleoli. Some cells were multiō nucleate. The appearances were those of a sarcoma probably a pleomorphic leiomyosarcoma. Staging investigations did not reveal any evidence of metastases outside the abdomen.

Whilst awaiting chemotherapy the tumour progressed over a period of one week and she developed features of subacute small bowel obstruction. She was therefore treated immediately with combination chemotherapy consisting of cyclophosphamide, adriamycin and vincristine with an excellent clinical response. Six cycles of this chemotherapy produced a complete remission on examination and CT scanning and a second-look laparotomy was planned. Unfortunately, whilst awaiting this operation there was rapid regrowth of the tumour requiring an urgent laparotomy and resection of deposits in the transverse colon and small bowel. Histological appearance of these tumours was the same as the primary tumour. One month later the disease progressed again and on 5 this occasion failed to respond to chemotherapy. She died with uncontrolled intra-abdominal sarcoma 10 months after her presentation.

At the age of 14 months the patient had suffered a scald which had required split skin grafting to the right $\overparen{\mathbb{D}}$ arm. Following this she became severely constipated, $\stackrel{\mathcal{D}}{\rightarrow}$ thought to be the result of the emotional trauma. She

(C) The Fellowship of Postgraduate Medicine, 1989 
was treated with the laxative Dorbanex (danthron with poloxalkol), $5 \mathrm{ml}$ orally at night and this treatment was continued until her fifth or sixth year. Regular treatment was then discontinued but she took Dorbanex intermittently for constipation throughout the rest of her life.

\section{Discussion}

In this case we have seen a very rare tumour in a child who had had prolonged exposure to danthron. This clearly is not sufficient evidence to postulate a causal relationship. However, the biological and experimental background suggests that the possibility of a causal relationship should be considered. The evidence that several anthraquinones are mutagenic in Salmonella typhimurium is strong. ${ }^{1,2}$ In rodents, the data are less extensive, with small numbers of animals, and they do not constitute conventional carcinogenicity studies. However, they do suggest a possible carcinogenic effect. Among 12 rats fed with large doses of danthron and surviving for one year, 4 developed adenocarcinoma of the large bowel; there were no tumours

\section{References}

1. Brown, J.P. \& Brown, R.J. Mutagenesis by 9,10anthraquinone derivatives and related compounds in Salmonella typhimurium. Mutation Res 1976, 40: 203-224.

2. Tikkanen, L., Matsushima, T. \& Natori, S. Mutagenicity of anthraquinones in the salmonella preincubation test. Mutation Res 1983, 116: 297-304.

3. Mori, H., Sugie, S., Niwa, K., Takahashi, M.\& Kawai, K. Induction of intestinal tumours in rats by chrysazin. $\mathrm{Br} J$ Cancer 1985, 52: 781-783. in 14 control rats observed for one year. ${ }^{3}$ Among 17 mice surviving for 500 days on large doses of oral danthron, 4 developed hepatocellular carcinomas and there were none in 19 control mice. In both treated and control mice, hepatic adenomas were seen. ${ }^{4}$ No evidence of sarcomas was reported. The mechanisms underlying these effects are unclear.

An epidemiological survey of 1,975 dye workers exposed to various anthraquinones showed no increased mortality from any cause. ${ }^{5}$ In our case, however, exposure was oral, prolonged and at an early age.

Anthraquinone laxatives have been widely used in children and adults for about 20 years and are still marketed. If they were found to be carcinogenic then it is possible that some resulting gastrointestinal tumours in young people might now be appearing. We are currently carrying out a pilot survey of young patients with gastrointestinal tumours to see if there is any further evidence for an association between danthron use and bowel cancer risk, and we would be extremely interested to hear of any similar cases diagnosed at other centres. If other anecdotal evidence can be found, we propose to institute a formal case-control study.

4. Mori, H., Sugie, S., Niwa, K., Yoshimi, N., Tanaka, T. \& Hiron, I. Carcinogenicity of chrysazin in large intestine and liver of mice. Jpn J Cancer Res (Gann) 1986, 77: 871-876.

5. Gardiner, J.S., Walker, S.A. \& Macleari, A.J. A retrospective mortality study of substituted anthraquinone on dye stuffs workers. Br J Indust Med 1982, 39: 355-360. 